\title{
A Mix Block Compressive Sensing Algorithm for Image Processing
}

\author{
Ruipeng Luan ${ }^{1, a}$ \\ ${ }^{1}$ The Chinese People's Liberation Army Unit 91550, Dalian, 116023, China \\ aemail: Irp_ph@163.com
}

Keywords: Mix Block; Compressive Sensing; Reconstruction; Image Processing

\begin{abstract}
In order to solve the problem of high cost and high time consuming in the traditional algorithm of compressed sensing technology, a mix block compressive sensing algorithm for image processing is proposed in this paper. Image is divided into sub images by mixed block strategy in the process of scanning, and then the sub images obtain parallel independent compression sampling. The proposed algorithm improves the sampling efficiency greatly, and provides a scheme to real time processing for compressive sensing. The effectiveness of the proposed algorithm is illustrated by the experimental data.
\end{abstract}

\section{Introduction}

Compressive sensing algorithm [1] [2] , combines sampling and compression, has the characteristic of direct information sampling, has advantageous to reduce the sampling cost and save the resources. The cost of the reconstruction algorithm of compressive sensing algorithm in big scale signal real-time sampling is very large. In order to solve these problems, Gan reference block DCT coding idea, put forward the block compressed sensing (BCS) [3], BCS algorithm uses the same measurement matrix to separate the sub images of the image, the independent compression sampling is carried out in parallel, improves the sampling efficiency greatly, and provides a scheme to real time processing for compressive sensing. In order to improve the performance of reconstruction, Mun [4] uses sparse representation of complex tight frames in reconstruction process of BCS. A hybrid sampling method based on the combination of random sampling and partial Fourier sampling is proposed by Romberg [5], can improve the sampling efficiency greatly, but hardware implementation is more difficult.

In order to solve the problem, a mix block compressive sensing algorithm for image processing is proposed in this paper. Image is divided into sub images by mixed block strategy in the process of scanning, and then the sub images obtain parallel independent compression sampling. The proposed algorithm improves the sampling efficiency greatly, and provides a scheme to real time processing for compressive sensing. At the same time, the block strategy ensures that the algorithm complexity does not change with the size of the image, which is suitable for processing high resolution image.

\section{Brief introduction of compressed sensing theory}

Compressed sensing theory mainly consists of the sparse representation of the signal, the measurement matrix and the reconstruction algorithm.

The sparsity of the signal is expressed by an orthogonal transformation of the baseline signal, expressed as the linear coefficient of for the vast majority of zero or absolute value is very small, with few big coefficients can be said the signal, that signal is sparse in the base, able to transform to the amount as the original signal, a simple expression.

The measurement matrix is a kind of information sampling method, which is different from other traditional compression process. It plays the role of the original signal in the compressed sensing theory. The design of the measurement matrix is directly related to whether the original signal can be included into the vector of the sampling value through the projection of the measurement matrix, and then the original signal can be reconstructed effectively. At the same time the design of the 
measurement matrix is also related to the amount of sampling value, namely the use of sampling resources. Therefore, the measurement matrix plays a key role in the theory of compressive sensing.

Signal reconstruction algorithm is compressed sensing theory of signal recovery algorithm that is the core of the theory, that the length obtained by a measurement matrix for the measurement of $M$ value vector $y$ by solving linear equations to reconstruct the length $N$ of the original signal $x$. Because of the existence of the compressibility or sparsity of the signal, it is possible to determine the existence of the solution. At the same time, the measurement matrix of the measurement matrix in the compressed sensing method provides a theoretical guarantee for the solution of the problem to be solved by the RIP property.

\section{Design of the MBCS}

Step1

Total number of pixels to be processed is $N=m \times m$, get $S$ square blocks for each block, number of pixels of each square blocks is $n \times n$. Block number $c=1$, $s n$ and block number $c$ meet the following relationships.

$$
\left\{\begin{array}{c}
s=4 \times 2^{c}+4 \\
n=m / 5 \times 2^{(c-1)}
\end{array}\right.
$$

Let $c=c+1$, if $\mathrm{n}>32$, block end.

Step2

The $l_{1}$ norm constrained optimization problem is modified as a gradient constraint, like TV(Total Variation) algorithm [6] [7].

$\min \operatorname{TV}(\boldsymbol{x}) \quad$ s.t. $\boldsymbol{\Phi} \boldsymbol{x}=\boldsymbol{y}$

Sparse base $\boldsymbol{\Psi}$ is a gradient sparse basis $\operatorname{TV}(\cdot), \operatorname{TV}(\boldsymbol{x})$ is the sum of gradient of image $x$.

$$
\mathrm{TV}(\boldsymbol{x})=\sum_{i=1}^{I-1} \sum_{j=1}^{J-1}\left|(\nabla \boldsymbol{x})_{i, j}\right|=\sum_{i=1}^{I-1} \sum_{j=1}^{J-1} \sqrt{\left(D_{h} \boldsymbol{x}\right)_{i, j}^{2}+\left(D_{v} \boldsymbol{x}\right)_{i, j}^{2}}
$$

$D_{\mathrm{h}} X$ represents horizontal gradient, $D_{\mathrm{v}} X$ represents vertical gradient.

$$
\begin{aligned}
& \left(D_{h} \boldsymbol{x}\right)_{i, j}=x_{i+1, j}-x_{i, j} \\
& \left(D_{v} \boldsymbol{x}\right)_{i, j}=x_{i, j+1}-x_{i, j}
\end{aligned}
$$

Step3

TV algorithm has high precision and robustness in solving the problem of image reconstruction, but its operation speed is slow. Because the image is divided into blocks, the dimension of the reconstruction algorithm is small, which can reduce the complexity of the algorithm and reduce the computation time. Therefore, this paper chooses the TV algorithm as the compressed sensing reconstruction algorithm. The problem represented by the formula (2) is converted to the following problem solving.

$$
\min \operatorname{TV}(\boldsymbol{x}) \text { s.t. } \boldsymbol{\Phi}_{\boldsymbol{H}} \boldsymbol{x}=\Phi \boldsymbol{x}+\Phi^{\prime} \boldsymbol{x}=\boldsymbol{y}
$$

Step4

Combine blocks to form a new measurement matrix.

$$
\boldsymbol{\Phi}=\left[\begin{array}{llll}
\boldsymbol{\Phi}_{B} & & & \\
& \boldsymbol{\Phi}_{B} & & \\
& & \ddots & \\
& & & \boldsymbol{\Phi}_{B}
\end{array}\right]
$$




\section{Experiment and Results}

In the MATLAB environment, test the paper method by using the standard gray scale image Lena $(1024 \times 1024,8$ bit). Table 1 shows the sampling rate $M / N=20 \%$, which $N$ is the signal length, $M$ is the measured value vector length, Lena in different mean filter template size $F$ under the peak signal to noise ratio PSNR (dB) and structural similarity SSIM [8] performance.

Tab.1. PSNR and SSIM of Lena in different $F(M / N=20 \%)$

\begin{tabular}{|c|c|c|c|c|}
\hline$F$ & 0 & 3 & 4 & 5 \\
\hline PSNR & 25.82 & 27.65 & 26.43 & 26.11 \\
\hline SSIM & 0.7980 & 0.9104 & 0.9022 & 0.9002 \\
\hline
\end{tabular}

As can be seen from the table, this chapter method in $F=0$ image reconstruction effect is the worst, PSNR is only $25.82 \mathrm{~dB}$, than PSNR $F=3(27.65 \mathrm{~dB})$ reduced by about SSIM, $2.5 \mathrm{~dB}$ index also correspondingly reduced by about 0.05 . In order to facilitate the description, remember the proposed method for BCS-TV $(\mathrm{F}=0)$, MBCS-TV $(\mathrm{F} \neq 0)$.

The value of $F$ has a great influence on the performance of reconstruction, and the performance of $F=3$ is the best. So the MBCS -TV method is $F=3$ in the future.

In order to compare the quality of restored image more directly, Figure 1 shows the difference image of Lena after histogram equalization.

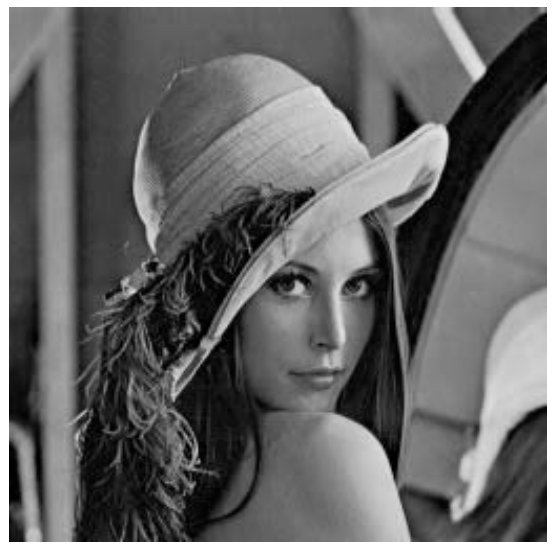

(a)

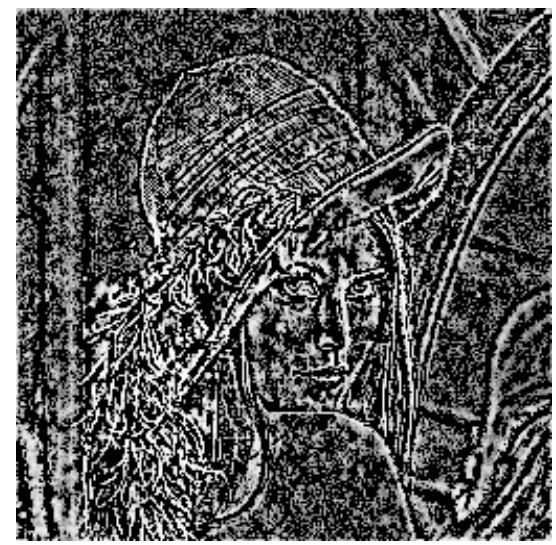

(b)

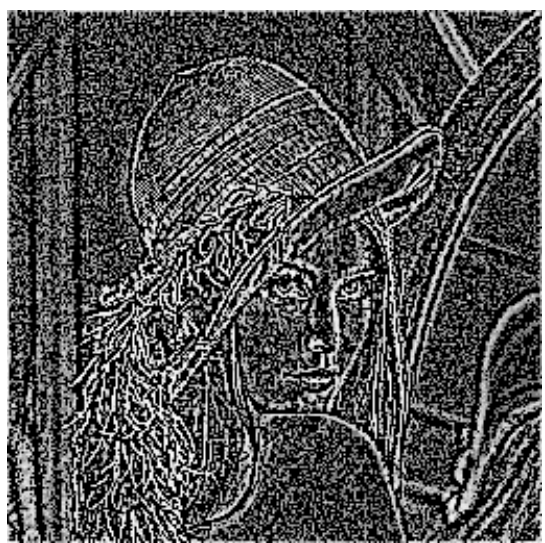

(c)

(a) Original image (b) $F=0(\mathrm{PSNR}=35.47 \mathrm{~dB}, \mathrm{SSIM}=0.8790)(\mathrm{c}) F=3(\mathrm{PSNR}=37.92 \mathrm{~dB}, \mathrm{SSIM}=0.9274)$

Fig.1. $M / N=20 \%$ difference image of Lena

As can be seen from Figure 1, the difference image MBCS-TV gray value changed in a small range, especially in the smoothing of the image area obviously to be lower than the values range BCS-TV difference image gray, also can see, MBCS-TV with more details of the recovery, BCS-TV the difference image texture details of difference image is more clear, fully shows that mixed sampling method can obtain than a single random sampling reconstruction performance higher.

The MBCS-TV method uses the block strategy, the measurement matrix does not change with the image size, the time and memory space occupied in the measurement process is very small. Under the conditions of the same resources, MBCS-TV can handle the image size is far greater than the CS-TV method, in general, the processing size of $512 \times 512$ or more images are suitable. Taking the MATLAB environment as an example, the $2.93 \mathrm{GHz}$ method is presented to measure the time of different sizes of Lena images in the Intel dual core CPU MBCS-TV environment. Table 2 shows the comparison of the measurement matrix of MBCS-TV and CS-TV in two ways of measuring the storage space.

As can be seen from Table 2, with the increase of the image size, CS-TV resource consumption has an exponential increase, while the MBCS-TV has been maintained at a lower level. Under the limited resources, for example only $2 \mathrm{G}$ of memory, when the image size is $256 \times 256$ pixels, the 
sampling rate, the measurement matrix in CS-TV the need storage space to 2G (element type is double type), indicating that $256 \times 256$ image size is the upper limit of CS-TV and MBCS-TV can 30M memory use $1024 \times 1024$ or larger image.

Tab.2. Measurement time of MBCS-TV for different size image

\begin{tabular}{|c|c|c|c|c|}
\hline $\begin{array}{c}\text { Measurement } \\
\text { time (s) }\end{array}$ & $256 \times 256$ & $512 \times 512$ & $1024 \times 1024$ & $2048 \times 2048$ \\
\hline MBCS-TV & 0.1594 & 0.4443 & 1.2975 & 6.5018 \\
\hline CS-TV & 2.0456 & 10.3922 & 38.4299 & 119.2571 \\
\hline
\end{tabular}

\section{Conclusion}

In order to solve the problem of the time cost of the compressive sensing reconstruction algorithm, a mix block compressive sensing algorithm (MBCS) for image processing is proposed in this paper. Image is divided into sub images by mixed block strategy in the process of scanning, and then the sub images obtain parallel independent compression sampling. The proposed algorithm improves the sampling efficiency greatly, and provides a scheme to real time processing for compressive sensing. At the same time, the block strategy ensures that the algorithm complexity does not change with the size of the image, which is suitable for processing high resolution image. Experimental results show that the proposed MBCS algorithm not only greatly reduces the computational complexity, but also can effectively improve the quality of reconstruction, which has an important significance for real-time processing CS.

\section{References}

[1] Davenport $\mathrm{M}$ and Duarte $\mathrm{M}$, et al. Compressed sensing: theory and applications, Cambridge University Press, 2011:1-68.

[2] E Candès. The restricted isometry property and its implications for compressed sensing[J]. Acadèmie des sciences , 2006, 346(I): 598-592.

[3] L Gan. Block compressed sensing of natural images[C]. Proceedings of IEEE DSP, Cardiff, UK, July 2007:403-406

[4] S Mun and Fowler J E. Block compressed sensing of images using directional transforms[C]. Proceedings of IEEE ICIP, Cairo, Egypt, November 2009:3021-3024.

[5] J Romberg. Imaging via Compressive Sampling[J]. IEEE Signal Processing Magazine. 2008.04. $14-20$.

[6] Willett R M, Harmany Z T, and Marcia R F. Possion image reconstruction with total variation regularization [C]. Proceedings of IEEE ICIP, Hong Kong, September 2010:4177-4180.

[7] Francois Mirallès, Julien Beaudry, et al. Laser scanning system for inspecting large underwater hydroelectric structures[J]. Journal of Electronic Imaging , 2012, vol.19(2), 023015:1-14.

[8] Willett R M, Harmany Z T, and Marcia R F. Possion image reconstruction with total variation regularization [C]. Proceedings of IEEE ICIP, Hong Kong, September 2014:4177-4180. 